\title{
Odorant specificity of three oscillations and the DC signal in the turtle olfactory bulb.
}

Ying-Wan Lam ${ }^{1,2}$, Lawrence B. Cohen ${ }^{1}$, and Michal R. Zochowski ${ }^{1,3,4}$

${ }^{1}$ Department of Cellular and Molecular Physiology, Yale University School of Medicine, New Haven, CT 06520 and the Marine Biological Laboratory, Woods Hole, MA 02543.

${ }^{2}$ Present address: Department of Neurobiology and Behavior, SUNY Stony Brook, Stony Brook, NY 11794.

${ }^{3}$ Center for Complex Systems, Warsaw School of Advanced Social Psychology, Warsaw, Poland.

${ }^{4}$ Present address: Department of Physics, University of Michigan, Ann Arbor, MI 48109.

Lawrence B. Cohen, lawrence.cohen@yale.edu

Address before Sept. 1:

MBL

7 MBL St.

Woods Hole, MA 02543

Address after Sept. 1:

Dept. of Cellular and Molecular Physiology

Yale University School of Medicine

333 Cedar St.

New Haven, CT 06520

Running title: Odorant specificity of olfactory bulb oscillations.

words in summary: 240; words in Introduction: 514; total number of words: 4969;

text pages: 11; figures: 7 ; tables: 1

Key Words: Terepene sp., voltage-sensitive dyes, optical recording, 
Abstract

The odor-induced population response in the in vivo turtle (Terepene sp.) olfactory bulb consists of three oscillatory components (rostral, middle, and caudal) that ride on top of a DC signal. In an initial step to determine the functional role of these four signals, we compared the signals elicited by different odorants. Most experiments compared isoamyl acetate and cineole; odorants which have a very different maps of input to olfactory bulb glomeruli in the turtle and a different perceptual quality for humans.

We found substantial differences in the response to the two odors in the rise-time of the DC signal and in the latency of the middle oscillation. The rate of rise for cineole was twice as fast as that for isoamyl acetate. Similarly, the latency for the middle oscillation was about twice as long for isoamyl acetate as it was for cineole.

On the other hand, a number of characteristics of the signals were not substantially different for the two odorants. These included the latency of the rostral and caudal oscillation, the frequency and envelope of all three oscillations and their locations and spatial extents. A smaller number of experiments were carried out with hexanone and hexanal; the oscillations elicited by these odorants did not appear to be different from those elicited by isoamyl acetate and cineole.

Qualitative differences between the oscillations in the turtle and those in two invertebrate phyla suggest that different odor processing strategies may be used. 
Following their discovery by Adrian (1942), odor stimuli have been shown to elicit or alter oscillatory events in the olfactory bulb in all species tested (e.g. Ottoson, 1959; Hughes \& Mazurowski, 1962; Beuerman, 1975; Laurent \& Naraghi, 1994, Dorries and Kauer, 2000). Using voltage-sensitive dye imaging, we found that the odorant-induced response in the olfactory bulb of the turtle actually consisted of four signals, three oscillations, named rostral, middle and caudal according to their location in the bulb, and a DC signal (Lam et al., 2000). The DC signal (Kauer, Senseman \& Cohen, 1987; Kauer, 1988), which occurs over the whole area of the bulb, has the shortest latency and lasts the longest. We carried out experiments to determine whether the properties of these four components are odorant dependent.

We used a 464 element photodiode array to monitor the activity in the in vivo turtle preparation. Each element of the array received light from a $170 \times 170 \mu \mathrm{m}^{2}$ area of the olfactory bulb and thereby monitors the membrane potential of a large number of neurons and neuronal processes. Any optical signal must represent changes in membrane potential that are at least partially synchronous (coherent) in some fraction of those neurons and processes. These membrane potential changes could arise from action potentials, synaptic potentials, or subthreshold oscillations in synaptically coupled neurons.

Hypotheses about the function of the olfactory bulb in odorant recognition can be broadly divided into two non-exc/usive categories. The first proposes that the identity of odorants are represented in the bulb by the spatial map of activity across the bulb (Stewart et al., 1979; Cinelli et al., 1995; Mori \& Yoshihara, 1995; Mombaerts et al., 1996; Friedrich \& Korsching, 1998; Wachowiak and Cohen, 2001; Wachowiak et al., 2002). The second suggests that the temporal structure of the neuronal response provides information about odorant identity (Mozell, 1964; Delaney et al., 1994; Hopfield, 1995; Laurent et al., 1996; Stopfer et al., 1997, PerezOrive et al., 2002). Laurent et al., (1996) showed that the envelope of the local field potential oscillations in the locust could be both complex and different for different odorants. Stopfer et al., (1997) found that disrupting the oscillations in the bee reduced their ability to make fine odor discriminations.

Physical processes occurring at the level of the olfactory epithelium were hypothesized to have differential effects on the time course of the response to different odorants (Mozell, 1966, Mozell and Jagodowocz, 1973; Kent et al., 1996). We provide a test of this hypothesis by examining the temporal characteristics of responses in the olfactory bulb.

In addition to the three oscillations found in the olfactory bulb, a fourth oscillation can be measured by extracellular recording from the olfactory epithelium; this oscillation is a component of the electroolfactogram (EOG). Evidence presented elsewhere (Ottoson, 1959; Dories and Kauer, 2000, Figure 3C; Lam et al., 2000; M. Zochowski and L. B. Cohen, unpublished observations) suggests that the oscillations in the EOG are only indirectly related to the oscillations seen in the olfactory bulb.

Abstracts of the results have been presented (Lam et al., 1998, 1999).

\section{MATERIALS and METHODS}


Preparation.

Three species of box turtle, Terepene carolina, T. ornata and T. triunguis were used. No species difference was seen. Most of the animals were obtained from Charles D. Sullivan Co., Inc. (Nashville, TN) and weighed between $280 \mathrm{~g}$ and $800 \mathrm{~g}$.

The composition of the turtle saline was: $\mathrm{NaCl}, 96.5 \mathrm{mM}, \mathrm{KCl}, 2.6 \mathrm{mM}, \mathrm{MgCl}_{2}, 2.0 \mathrm{mM}, \mathrm{NaHCO}_{3}, 31.5$ $\mathrm{mM}, \mathrm{CaCl}_{2}, 4.0 \mathrm{mM}$, Dextrose, $10 \mathrm{mM}$. The saline was bubbled with $95 \% / 5 \% \mathrm{O}_{2} / \mathrm{CO}_{2}$ to $\mathrm{pH} 7.2$ before using. These chemicals were obtained from Sigma.

Details of the surgical procedures have been described previously (Lam et al., 2000). Briefly, the turtles were first anesthetized by placing them in ice for 2 hours. Shortly before surgery, lidocaine $(0.4$ to $0.6 \mathrm{ml}, 1 \%$ w/v solution in saline) was applied near the site of craniotomy as a local anesthetic and tubocurarine ( $3 \mathrm{mg} / \mathrm{kg})$ was injected intra-peritoneally or intra-muscularly to partially paralyze the animals. A craniotomy was performed over the olfactory bulb. To facilitate staining, the dura and arachnoid mater were then carefully removed. After the dissection, a segment of polyethylene tubing of appropriate length and diameter (OD 2.0 $\mathrm{mm}$, ID $1.0 \mathrm{~mm}$ ) was inserted into the outlet of the nasal cavity in the roof of the mouth to facilitate odorant delivery. The experimental protocol was approved by the Yale Animal Care and Use Committee and the Marine Biological Laboratory Institutional Animal Care and Use Committee.

\section{Odorant delivery.}

The design of the olfactometer was copied from Kauer \& Moulton (1974). Cleaned and desiccated carrier gas, air with $1 \% \mathrm{CO}_{2}$, and laboratory air saturated with odorant vapor were mixed in the inner tube of a double-barrel odorant-applicator. The concentration of the odorant was adjusted by changing the flow-rate of the odorant vapor. The outer tube of the applicator was normally under suction to remove the odorant but at a command pulse, this suction was turned off to release a square-pulse of odorant. The horizontal bars in Figures 1-6 indicate the timing of this command pulse. Suction (100-300 $\mathrm{ml} / \mathrm{min})$, controlled by a separate, independent solenoid valve, was applied on the segment of tubing inserted into the nasal outlet. This suction was switched on 1-2 seconds before the odorant-pulse and continued for 15-20 seconds to draw the air - then odorant -then air into the nasal cavity. For additional details see Lam et al., (2000).

\section{Odorants.}

The odorants were obtained from Sigma. The concentrations of odorant were $0.3 \%, 1.7 \%, 2.5 \%, 10 \%$, and $15 \%$ of saturated vapor. For statistical comparisons the trials with $10 \%$ and $15 \%$ were grouped together as the high concentration condition and the trials with $1.7 \%$ and $2.5 \%$ were grouped together as the low concentration condition.

\section{Optical Imaging.}

The dorsal olfactory bulb (about $30 \%$ of the total bulb surface area) was imaged using a macroscope designed to provide a large numerical aperture at low magnification. The macroscope was based on a $25 \mathrm{~mm}$ focal length, $0.95 \mathrm{f}$, camera lens (RedShirtImaging, LLC, Fairfield, CT). The preparation was illuminated using a $100 \mathrm{~W}$ tungsten halogen lamp. The excitation filter was $520 \pm 45 \mathrm{~nm}$. A $590 \mathrm{~nm}$ long-pass dichroic mirror 
(Omega Optical, Brattleboro, VT) reflected the excitation light onto the preparation. The secondary filter was a RG610 long-pass filter (Schott Optical Glass, Duryea, PA). For earlier experiments a 464 element photodiode array (Centronics, Co, New Addington, England) was used. In later experiments we used NeuroPlex, a 464element light-guide and photodiode imaging system (RedShirtlmaging, LLC). At the $4.5 \times$ magnification used each element of the array received light from a $170 \times 170 \mu \mathrm{m}$ area of the object plane. The cut-off of the singlepole RC high-pass filter of each amplifier was set to $0.07 \mathrm{~Hz}$ and the low-pass cut-off of the 4-pole switched capacitance Bessel filter was $125 \mathrm{~Hz}$. The data acquisition rate was 250 frames per second. The light intensity reaching each photodetector was approximately $2 \times 10^{8}$ photons $/ \mathrm{msec}$ which generated a photocurrent of about $5 \times 10^{-8}$ amperes. All of the results come from single trials; signal averaging could not be used because the timing of the osciallations were not precisely locked to the odorant onset. Higher-resolution pictures of the preparations were taken with a CCD camera (MTI RC300, Michigan City, IN). The images taken with the photodiode array and the CCD camera were aligned using a 3-dot calibration pattern.

\section{Staining.}

The exposed olfactory bulb was stained by covering it with a solution of the styryl dye RH414 (Grinvald et al., 1994; Molecular Probes, T-1111) 0.01 to $0.2 \mathrm{mg} / \mathrm{ml}$ in saline for 60 minutes. After staining, excess dye was washed away with turtle saline. Examination of the sectioned bulb at the end of experiments showed that the staining appeared uniform throughout the thickness of the exposed portion of the bulb.

\section{Data Analysis.}

The NeuroPlex software was used to digitally filter and display the data. The high-pass filter was a numerical-simulation of an RC circuit and the low-pass filter was a Gaussian. The fractional change in fluorescence, $\Delta F / F$, was calculated and plotted as the traces in Figures 1-6. The signal sizes ranged from $10^{-4}$ (middle oscillation) to $5 \times 10^{-3}(\mathrm{DC})$ of the total intensity. Voltage-sensitive dye measurements on turtle olfactory nerves were used to determine the sign of an optical signal that represents a membrane depolarization; signals in the bulb that are inferred to represent depolarization are plotted upward. Two different schemes were used for assigning colors in pseudocolor presentations, fixed and variable. For the fixed pseudocolor display, the diode with the largest signal was found by visual inspection. Red color represents a signal size which exceeds $75 \%$ of the size of the largest signal. This scale was then used to assign colors to the signals on all other detectors. This kind of pseudocolor presentation represents the relative size of the signals and was used in all the figures except for the bottom two panels in Figure 7. For the variable pseudocolor display the peak signal for each detector was assigned red and the nadir assigned purple; in this way every detector reaches red at some time during the recording independent of its signal size. This variable pseudocolor scale correctly represents the relative timing of the signals but it is susceptible to noise from detectors with small signal-tonoise ratios.

The latency of the DC signal was calculated from the time difference between the onset of the odorant command pulse (Figure 2, red arrowheads) and start of the DC component (Figure 2, black arrowheads). The rise-time (10\%-90\%) of the DC component was measured from the traces. To analyze the oscillations, the 
traces were band-passed filtered at $10-30 \mathrm{~Hz}$; this converts the DC component into an initial peak (e.g. Figure $1, \mathrm{DC}$ ) or, in instances where the rise-time is slow, can remove any sign of the DC component (e.g. Figure 5, bottom traces). The latency of an oscillation was defined as the time difference between the start of the odorant command pulse and the first peak of the oscillation (Figure 3, black arrowheads). The initial frequency of an oscillation was estimated from the time difference between the first peak (Figure 3, black arrowheads) and the third peak (Figure 3, red arrowheads).

In Table 1 we used paired t-tests to compare the rise-time and latency of the DC signals induced by cineole and isoamyl acetate. We first selected preparations that had DC responses with relatively large signalto-noise ratios to both odorants at matching concentrations (9 out of 37 preparations). For each animal, the trials with the same odorant type and concentration were grouped together and the average rise-time and latency of each group was computed. Paired t-tests were then used to analyze these averages. Effects of the odorant at high ( $15 \%$ or $10 \%$ of saturation) and low $(2.5 \%$ or $1.7 \%)$ concentration are separately analyzed and presented. The statistical procedures for analyzing the latency and initial frequency of the rostral and middle oscillation for Table 1 were the same. Because only four animals were useful in the analysis of the caudal oscillation, the responses to the same odorant at both high and low concentration needed to be combined to generate a group large enough for use in comparisons.

Three odorants, cineole, isoamyl acetate, and hexanal, were tested in four additional preparations. We used repeated-measure ANOVA, one-way ANOVA and post-hoc analysis with Fisher's PSLD to analyze these experiments.

\section{RESULTS}

The three traces on the bottom of Figure 1A, taken from data published previously (Lam et al., 2000), show examples of the three oscillations, rostral, middle and caudal, and their location on the turtle olfactory bulb (Figure 1A, top). In addition to location, the oscillations differ in frequency, latency, shape, and time course of the envelope of the oscillation. Furthermore, all three traces in Figure 1 have a filtered version of the DC signal at the time indicated by the bar labeled DC (filtered); the DC signal is detected over the entire dorsal bulb. We made relatively extensive comparisons of the signals elicited by two odors, isoamyl acetate and cineole, which have a very different perceptual quality for humans (Arctander, 1994). Furthermore, these two odorants have qualitatively different maps of input to the turtle olfactory bulb as indicated by measurements of Calcium Green fluorescence from olfactory receptor neuron terminals (Figure 1B; Wachowiak et al., 2002). The figure illustrates maps of the input for two concentrations of isoamyl acetate (top) and cineole (bottom). The maps for the two odorants are qualitatively different at both concentrations. In addition to isoamyl acetate and cineole, a smaller number of experiments were carried out with hexanal and hexanone.

Figure 2 illustrates typical examples of the DC component and the middle oscillation elicited by cineole and isoamyl acetate. Traces from the middle part of the olfactory bulb are shown. The red and black arrowheads on the traces indicate where we designated the onset of the odorant and the onset of the DC 
response. The response elicited by $1.7 \%$ isoamyl acetate rises much more slowly (Figure $2 \mathrm{~A}$ ) than the response to $1.7 \%$ cineole (Figure $2 \mathrm{~B}$ ). In measurements made at odorant concentrations that were six times higher, cineole had a similarly faster rise-time. To quantitate the differences we measured the rise-time (10\%$90 \%$ ) of the DC signals. The isoamyl acetate DC signal had a rise-time that was about twice as long as that for cineole; statistical analysis showed that the difference was significant, $p<0.001$ (Table 1). The pseudocolor images in Figure 2B and D illustrate the spatial spread of the DC signal (at substantially different frame intervals).

The middle oscillation can be seen riding on top of the DC response (three blue arrowheads in Figure 2). To show the middle oscillation more clearly, the data of Figure 2 are illustrated after high-pass filtering to remove low-frequency components and increasing the $y$-axis gain (Figure 3A, C). The latency of the middle oscillation (indicated by the black arrowheads) elicited by isoamyl acetate was substantially longer than that elicited by cineole. (If an oscillation begins as a small signal, the latency determination will be imprecise. As a result we attempted to be conservative with regard to concluding that there a difference between cineole and isoamyl acetate. For example, the start of the middle oscillation in response to cineole was chosen conservatively (black arrow, Figure $3 \mathrm{C}$ ); the arrow could have been positioned at the earlier peak on the falling phase of the DC signal. Because of this imprecision in determining the latency, only large latency differences are considered.) Two additional comparisons illustrating a shorter latency for cineole are shown in the top portion of Figure 6 . The mean latency of the middle oscillation in response to cineole was 200 msec shorter than that of isoamyl acetate; $p<0.05$ (Table 1). The percentage differences in the latencies given in Table 1 (30-35\%) are an underestimate because we took as the starting time the beginning of the command pulse to the solenoid controlling the odorant delivery and there is a substantial ( $100 \mathrm{msec})$ delay between the start of the command pulse and the arrival of odorant at the olfactory epithelium. If the beginning of the DC signal were used as the starting point for the latency measurements, then the percentage difference would be larger.

The difference in latencies between cineole and isoamyl acetate was specific to the middle oscillation. In another trial from the same animal in which both the middle and the rostral oscillation were detected, the middle oscillation induced by isoamyl acetate again had a longer latency (arrows, Figure 4A); however, in contrast, the rostral oscillation in response to isoamyl acetate and cineole had similar latencies (arrows, Figure 4B). The grouped data also showed no significant differences in the latency of the rostral oscillations evoked by cineole and isoamyl acetate; $p=0.48$ (Table 1 ).

Table 1 also summarizes the comparisons of the initial frequency of the three oscillations using paired t-tests. Odorant type also had no effect on the initial frequency of the middle oscillation or the caudal oscillation ( $p>0.3$ ). In this comparison odorant type had a small, $5 \%$, but significant effect on the initial frequency of the rostral oscillation, $p<0.05$. The measurements for the rostral oscillation were only made at high odorant concentration; there was not enough data at low concentration.

Repeated trials in one preparation. The above statistical analyses considered the means for the two odorants for each preparation and then compared these means across preparations. To examine trial-to-trial 
variability in individual preparations we compared the individual trials in preparations where there were three or more trials for both cineole and isoamyl acetate. For the rise-times of the DC signal we performed unpaired t-tests in five preparations. The differences were significant in four preparations $(p<0.005)$; the difference was not significant in one $(p=0.065)$. The number of trials ranged from 9 to 21 . For the latency of the middle oscillation we performed unpaired t-tests in two preparations; the differences were significant in both $(p<$ 0.001). The number of trials that were compared was 12 and 16. Thus, the differences in DC rise-time and latency of the middle oscillation were also significant in trial-to-trial comparisons in individual preparations.

Comparison with hexanal. In four animals we compared several parameters of the response to hexanal, cineole, and isoamyl acetate. The rise-time (10\%-90\%) of the DC signals were compared using repeatedmeasure ANOVA. The differences between odorants was statistically significant $(F=5.71, p<0.05)$. Post-hoc analysis using Fisher's PSLD indicated that the differences between cineole and isoamyl-acetate, and cineole and hexanal were significant $(p<0.05)$. The difference between isoamyl-acetate and hexanal was not significant $(p>0.5)$. There is a similar relationship between the three odorants in the location of their input to the olfactory bulb; the maps of input were similar for hexanal and isoamyl acetate but different for cineole (Wachowiak et al., 2002).

Comparison of the rostral oscillation for the three odorants using repeated-measure ANOVA showed, in contrast to the small effect seen comparing only cineole and isoamyl acetate (see above), that the latencies and initial frequency were not significantly different across odors ( $p>0.05$ ); e.g. Figure 5, bottom. The caudal oscillation could be compared in only two of the preparations. The trial-to-trial differences of the frequency and latency was measured using one-way ANOVA. Neither the latency nor the frequency of the caudal oscillations were different for the three odorants.

\section{Envelope of the oscillations.}

In the locust, different odorants evoke local field potential oscillations that can have reproducibly different envelopes (Figure 5, Laurent et al., 1996; G. Laurent, personal communication). In contrast, Figure 5 shows three comparisons of the rostral oscillation (including four odorants) and Figure 6 shows two comparisons of middle oscillations (two odorants), and two comparisons of caudal oscillations (three odorants). In all instances the envelopes of the oscillations appeared to increase and decrease monotonically. Furthermore, in contrast to the results in the locust, in all seven comparisons in Figures 5 and 6 the envelopes of the oscillations are very similar. This is the typical result in those instances where the two odorants evoke oscillations of approximately equal amplitude.

\section{Location and areal extent.}

The areal extent of the four components was always much larger than the area of a single glomerulus even at odorant concentrations as low as $0.3 \%$ (Figures 2, 3, and 7). Comparisons of the locations of the DC component and the middle oscillation elicited by cineole and isoamyl acetate at $1.7 \%$ of saturation are shown in the pseudocolor images in Figures 2 and 3. The position of the DC signal during the rising phase (times indicated by red lines) is shown in Figure $2 B$ and $2 \mathrm{D}$. The $\mathrm{DC}$ components elicited by both odorants started in 
the middle-caudal bulb and then spread over a large area of the bulb. Figure 2B and D shows only the signals that are $>60 \%$ of the largest signal. Smaller DC signals are seen over the remaining area of the bulb. Clearly, the areas of activation of the DC component for the two odorants overlap extensively. Although the locations of the initiation sites and the shapes of the activated areas differed slightly in this example, these differences were not consistent across trials. The locations of the middle oscillation during one cycle (indicated by red lines) are shown in Figure 3B and D. The locations of the middle oscillations elicited by cineole and isoamyl acetate were not detectably different. They both started in the middle-caudal region of the bulb and then spread to a substantial portion of the caudal-middle area.

Figure 7 compares the extent and locations of the three oscillations using an expanded presentation of a single frame taken at the peak of one cycle of an oscillation. The top three frames of Figure 7A show the location of the rostral oscillation in response to $0.3 \%$ cineole, isoamyl acetate, and hexanone; the rostral oscillation has the same location for all three odorants. Similar comparisons between cineole and isoamyl acetate were made in 15 pairs of trials from 10 preparations (odorant concentration range 0.3 to $15 \%$ ); in all cases the rostral oscillations for the two odorants were found in apparently identical locations. In other experiments the rostral oscillations in response to all four odorants (cineole, isoamyl acetate, hexanal, and hexanone) had the same location and extent (data not shown). Figure 7B compares the propagation of one cycle of the rostral oscillation for $0.3 \%$ cineole and isoamyl acetate. The rate and direction of propagation for the two different odorants is similar.

Figure 7C illustrates an additional comparison of the location of the middle oscillations for cineole and isoamyl acetate. Again the location of this oscillation is apparently identical for the two odorants. Similar results were obtained in three other preparations. Figure 7D compare the locations of the caudal oscillation in response to cineole and isoamyl acetate. While the example indicates that the location of the two caudal oscillations is identical, this result must be qualified because the location of the caudal oscillation changes from cycle-to-cycle (Figure 6 of Lam et al., 2000). Thus, in other cycles from the same trial the location may change by up to 3 pixels on the photodiode array $(\sim 450 \mu \mathrm{m})$. Similar comparisons for the caudal oscillation were made in 10 pairs of trials from 7 preparations (odorant concentration range 1.7 to 15\%); in no case were the differences in the locations of the caudal oscillation for cineole and isoamyl acetate larger than the cycle-tocycle differences.

\section{DISCUSSION}

We compared the population responses in the turtle olfactory bulb elicited by four odorants with differing functional groups (an acetate, an aldehyde, a ketone, and a heterocyclic molecule); the most complete data compares cineole and isoamyl acetate. These two odorants are perceptually quite different to humans and have qualitatively different maps of input to olfactory bulb glomeruli in the turtle. We found that two temporal parameters of the signals were substantially different for cineole and isoamyl acetate: the rise-time of the DC component was faster and the latency of the middle oscillation was shorter for cineole (Table 1). This 
latency difference cannot be explained by a general speed-up of the responses to cineole because the latencies to the rostral and caudal oscillations were not different for cineole and isoamyl acetate.

In one comparison of the initial frequency of the rostral oscillation, we found a small $(5 \%)$ difference that was significant (Table 1); in a second comparison (including hexanal) a significant difference was not detected. Both Hughes and Mazurowski (1962) in the monkey and Dorries and Kauer (2000) in the salamander reported small odorant dependent differences in olfactory bulb oscillation frequency.

The remaining parameters that we measured appeared to be the same for the odorants we tested. These include the location, envelope, and areal extent of all three oscillations, the frequency of the middle and caudal oscillation, and the propagation direction and velocity of the rostral oscillation.

\section{Rate of rise of the DC component.}

We found a dramatic difference in the rate of rise of the DC component for cineole and isoamyl acetate; the rise-time of the response to isoamyl acetate was almost two times slower than that of cineole. Mozell and Jagodowicz (1973) measured the difference in latency of the response of two branches of the olfactory nerve in the frog and compared that to the difference in retention time by the olfactory mucosa. They found that odorants with low retention times on vapor phase chromatography (Fuller et al., 1964) had small differences in latency between the two branches of the olfactory nerve. From this result one might expect that odorants with lower retention times would result in more synchronous activation of receptors and thus more rapid DC signals. We found the opposite result. Amyl acetate has a retention time that is about one half that of cineole and thus would be expected to have a faster response time. Apparently other factors must be important in determining the rate of rise. These factors might include differences in rates of diffusion in the olfactory mucosa or differences in the range of odorant thresholds in the receptor neurons activated by the two odorants.

\section{Large and smooth spatial extent.}

The spatial extent of the three oscillations was large at all of the odorant concentrations we tested $(0.3$ $\%$ to $15 \%$; Figures 2, 3, 7). Furthermore, the spatial maps of the oscillations do not have the individual glomerular detail that has obtained using Calcium Green to record the activity of olfactory receptor axon terminals (Friedrich and Korsching, 1997; Wachowiak and Cohen, 2001; Wachowiak et al., 2002) or from intrinsic imaging signals (Rubin and Katz, 1999; Meister and Bonhoeffer, 2000; Uchida et al., 2000). We presume that the present results, obtained after staining all of the neurons in the bulb with the voltage sensitive dye, reflect the activity of many neuron types throughout the thickness of the bulb. Although it is well known that axonal projections follow a precise spatial map to individual glomeruli (Mombaerts et al., 1996), it is not clear that olfactory processing that occurs in the bulb maintains the same spatial precision. Our data suggests a spatially distributed processing in the olfactory bulb. Additional measurements using staining methods that specifically label the mitral/tufted neurons would be required to determine whether the output of the bulb is similarly distributed.

\section{Temporal codes in the turtle.}

Our results are not inconsistent with the use of temporal encoding for odorant recognition in the turtle 
but a strong conclusion cannot be made. On one hand, we did find substantial odorant dependent differences in the rise-time of the DC signal and in the latency for the middle oscillation. In addition, we previously reported that the caudal oscillation shows cycle to cycle differences in location (Lam et al., 2000), reminiscent of the differing neuronal associations that occur from cycle to cycle in the locust (Laurent et al., 1996). On the other hand, we did not detect substantial odorant dependent differences in the frequency, envelope, or spatial extent of any of the oscillations. One possibility is that these oscillation signals are used to code for something other than odor quality.

\section{Comparison with oscillations in invertebrates and other vertebrates.}

Olfactory processing in arthropods and molluscs may differ qualitatively from that found in the turtle. We found no odorant dependent differences in envelopes of the three oscillations (Figures 5-6). (We are not aware of evidence concerning the odorant dependence of the envelope of the oscillations in other vertebrates.) In contrast, substantial odorant dependent differences in the oscillation envelopes can be seen in the locust (Figure 5, Laurent et al., 1996; G. Laurent, personal communication). Perez-Orive et al (2002) suggest that the oscillations in the locust contribute to sparsening of odorant representation in the antennal lobe. The fact that the oscillation envelopes in the turtle are apparently independent of odorant allows the speculation that their role in olfactory processing may not be related to odor recognition.

In the mollusc it appears that yet another scheme is used. There is a steady-state $\sim 1 \mathrm{~Hz}$ oscillation that is a propagating wave across the procerebral lobe. This oscillation is present in the absence of odor and its frequency and/or propagation velocity is altered by air and odorants (Delaney et al., 1994, Nikitin and Balaban, 2000).

These differences between molluscs, insects, and the turtle suggests that, even though oscillations are widely observed in olfactory processing, the functional roles of these oscillations may vary; diverse strategies for olfactory processing may be used in different phyla. 
Acknowledgments: We are grateful to David Senseman for the loan of the 464 element photodiode array and to John Kauer for help with the construction of the olfactometer. We thank Barbara Ehrlich, Brian Salzberg, Matt Wachowiak, and Dejan Zecevic for their helpful comments on the manuscript. Supported in part by $\mathrm{NIH}$ grant DC05259 and a Brown-Coxe fellowship from the Yale University School of Medicine. 


\section{REFERENCES.}

Adrian, E. D. (1942). Olfactory reactions in the brain of the hedgehog. Journal of Physiology, 100, 459-473.

Arctander, S., (1994) Perfume and Flavor Chemicals (Aroma Chemicals), Allured Publishing, Carol Stream, IL.

Beuerman, R. W. (1975). Slow potentials of the turtle olfactory bulb in response to odor stimulation of the nose. Brain Research, 97, 61-78.

Cinelli, A. R., Hamilton, K. A. \& Kauer, J. S. (1995). Salamander olfactory bulb neuronal activity observed by video rate, voltage-sensitive dye imaging. III. Spatial and temporal properties of responses evoked by odorant stimulation. Journal of Neurophysiology, 73, 2053- 2071.

Delaney KR, Gelperin A, Fee MS, Flores JA, Gervais R, Tank DW, Kleinfeld DJ, (1994) Waves and stimulusmodulated dynamics in an oscillating olfactory network. Proc Natl Acad Sci U S A 91, 669-73.

Dorries K, Kauer, J., (2000) Relationships between odor-elicited oscillations in the salamander olfactory epithelium and olfactory bulb. J. Neurophysiology, 83, 754-765.

Friedrich, R. W. \& Korsching, S. I. (1997). Combinatorial and chemotropic odorant coding in the zebrafish olfactory bulb visualized by optical imaging. Neuron, 18, 737-752.

Fuller, G. H., R. Steltenkamp, and G. A. Tisserand (1964) The gas chromatograph with human sensor: perfumer model. Ann. N.Y. Acad. Sci. 116, 711-724

Grinvald, A., Lieke, E. E., Frostig, R. D Hildesheim, R. (1994). Cortical point-spread function and long-range lateral interactions revealed by real-time optical imaging of Macaque monkey primary visual cortex. Journal of Neuroscience, 14, 2545-2568.

Hopfield, J. J. (1995). Pattern recognition computation using action potential timing for stimulus representation. Nature, 36, 33-36.

Hughes, J.R. and Mazurowski, J. A. (1962) Studies on the supracallosal mesial cortex of unanesthetized, conscious mammals. II. Monkey. C. Frequency analysis of responses from the olfactory bulb. Electroenceph. 
Clin. Neurophysiol., 14, 646-653.

Kauer, J. S. (1988). Real-time imaging of evoked activity in local circuits of the salamander olfactory bulb. Nature, 331, 166-168.

Kauer, J. S. \& Moulton, D. G. (1974). Responses of olfactory bulb neurones to odour stimulation of small nasal areas in the salamander. Journal of Physiology, 243, 717-737.

Kauer, J. S., Senseman, D. \& Cohen, L. B. (1987). Odor activity monitored simultaneously from 124 regions of the salamander olfactory bulb using a voltage sensitive dye. Brain Research, 418, 255-261.

Kent, P. F., Mozell, M. M., Murphy, S. J., and Hornung, D.E. (1996) The interaction of imposed and inherent olfactory mucosal activity patterns and their composite representation in a mammalian species using voltage sensitive dyes. J. Neuroscience, 16, 345-353.

Lam, Y.-W., Cohen, L. B., Wachowiak, M. \& Zochowski, M. R. (2000). Odors elicit three different oscillations in the turtle olfactory bulb. J. Neuroscience. 20, 749-762.

Lam Y-W, Cohen L.B., Zochowski M (1998) Spatio-temporal properties of odor elicited responses in turtle olfactory bulb. Soc Neurosci Abs, 24, 28.

Lam, Y.-W., L. B. Cohen, M. Wachowiak, and M. Zochowski, (1999b). Spatio-temporal properties of odor responses in the turtle olfactory bulb. Abs Soc Neuroscience, 25, 126.

Laurent G, Naraghi M., (1994). Odorant-induced oscillations in the mushroom bodies of the locust. J Neuroscience 14, 2993-3004.

Laurent, G., Wehr, M. \& Davidowitz, H. (1996). Temporal representations of odors in an olfactory network. J. Neuroscience, 16, 3837-3847.

Meister, M. and Bonhoeffer, T. (1999) Optical recording of odorant responses from rat olfactory bulb. Soc Neurosci Abs, 25, 1050.

Mombaerts, P., Wang, F., Dulac, C., Chao, S. K., Nemes, A., Mendelsohn, M., Edmondson, J. \& Axel, R. (1996). Visualizing an olfactory sensory map. Cell, 87, 675-86. 
Mori K. \& Yoshihara, Y. (1995). Molecular recognition and olfactory processing in the mammalian olfactory system. Progress in Neurobiology, 45, 585-620.

Mozell, M. M. (1966). The spatiotemporal analysis of odorants at the level of the olfactory receptor sheet. J. General Physiology, 50, 25-41.

Mozell, M. M. and Jagodowicz, M. (1973). Chromatographic separation of odorants by the nose: Retention times measured across in vivo olfactory mucosa. Science, 181, 1247-1249.

Nikitin ES, Balaban PM, (2000) Optical recording of odor-evoked responses in the olfactory brain of the naive and aversively trained terrestrial snails. Learning and Memory, 7, 422-32.

Ottoson, D. (1959). Comparison of slow potentials evoked in the frog's nasal mucosa and olfactory bulb by natural stimulation. Acta Physiologica Scandinavica, 47, 149-159.

Perez-Orive, J., Mazor, O., Turner, G. C., Cassenaer, S., Wilson, R. I., and Laurent, G. (2002) Oscillations and sparsening of the odor representation in the mushroom body. Science, 297, 359-365.

Rubin, B.D., Katz, L.C. (1999) Optical imaging of odor representations in the mammalian olfactory bulb. Neuron, 23, 499-511.

Stewart, W. B., Kauer, J. S. \& Shepherd, G. M. (1979). Functional of rat olfactory bulb analysed by the 2deoxyglucose method. J. Comparative Neurology, 185, 715-734.

Stopfer, M., Bhagavan, S., Smith, B. H. \& Laurent, G. (1997). Impaired odour discrimination on desynchronization of odour-encoding neural assemblies. Nature, 390, 70-73.

Stopfer M, and Laurent G. (1999) Short-term memory in olfactory network dynamics. Nature, 402, 664-668.

Uchida, N., Takahashi, Y. K., Tanifuji, M., and Mori, K. (2000) Odor maps in the mammalian olfactory bulb: domain organization and odorant structural features. Nature Neuroscience, 3, 1035-1043.

Wachowiak, M., and Cohen, L. B., (1999), Presynaptic inhibition of primary olfactory afferents mediated by different mechanisms in the lobster and turtle. J. Neuroscience, 19, 8808-8817.

Wachowiak M and Cohen LB., (2001) . Representation of odorants by receptor neuron input to the mouse 
olfactory bulb. Neuron, $\mathbf{3 2}, 725-737$.

Wachowiak M, Cohen LB, and Zochowski, M. (2002) Distributed and concentration invariant spatial representations of odorants by receptor neuron input to the turtle olfactory bulb. J. Neurophysiology, 87, 10351045 . 
Table 1. Statistical analysis of parameters for the four components for isoamyl acetate and cineole.

\begin{tabular}{|c|c|c|c|c|c|c|c|}
\hline Component & Parameter & Concentration & Value for iaa 1 & Diff2 & $\%$ Diff 3 & $t$ (DF) & $\mathbf{p}$ \\
\hline \multirow[t]{4}{*}{$\mathrm{DC}$} & latency & high & $285 \mathrm{~ms}$ & $+14 \mathrm{~ms}$ & 5 & $0.74(9)$ & 0.48 \\
\hline & & low & $266 \mathrm{~ms}$ & $+8 \mathrm{~ms}$ & 3 & $0.55(9)$ & 0.59 \\
\hline & rise-time & high & $354 \mathrm{~ms}$ & $-141 \mathrm{~ms}$ & 40 & $6.6(6)$ & $0.0006^{*}$ \\
\hline & & low & $460 \mathrm{~ms}$ & $-188 \mathrm{~ms}$ & 41 & $5.9(7)$ & $0.0006^{*}$ \\
\hline \multirow[t]{2}{*}{ Rostral } & latency & high & $761 \mathrm{~ms}$ & $+46 \mathrm{~ms}$ & 6 & $0.74(7)$ & 0.48 \\
\hline & frequency & high & $14.9 \mathrm{~Hz}$ & $-0.7 \mathrm{~Hz}$ & 5 & $-2.8(7)$ & $0.027^{*}$ \\
\hline \multirow[t]{4}{*}{ Middle } & latency & high & $668 \mathrm{~ms}$ & $-233 \mathrm{~ms}$ & 35 & $-3.9(4)$ & $0.018^{*}$ \\
\hline & & low & $628 \mathrm{~ms}$ & $-200 \mathrm{~ms}$ & 32 & $-5.2(3)$ & $0.015^{*}$ \\
\hline & frequency & high & $14.4 \mathrm{~Hz}$ & $+1.2 \mathrm{~Hz}$ & 8 & $0.94(4)$ & 0.40 \\
\hline & & low & $13.3 \mathrm{~Hz}$ & $-.03 \mathrm{~Hz}$ & 2 & $-0.46(3)$ & 0.68 \\
\hline \multirow[t]{2}{*}{ Caudal } & latency & high/low & $1029 \mathrm{~ms}$ & $+186 \mathrm{~ms}$ & 18 & $-2.56(4)$ & 0.06 \\
\hline & frequency & High/low & $6.0 \mathrm{~Hz}$ & $-0.3 \mathrm{~Hz}$ & 5 & $0.99(4)$ & 0.38 \\
\hline
\end{tabular}

* Significant

1 Mean values for isoamyl acetate.

2 A positive number indicates that the response elicited by cineole has a larger parameter value, $M_{C}-M_{I A A}$ where $M_{C}$ and $M_{I A A}$ are the means for the response of cineole and isoamyl acetate.

$3\left(\mathrm{M}_{\mathrm{C}}-\mathrm{M}_{\mathrm{IAA}}\right) / \mathrm{M}_{\mathrm{IAA}}$.

4 The responses to the high and low concentration odorants were combined in this analysis. 
Figure 1. A. The time course and locations of the three oscillations that occur in response to odorants in the turtle olfactory bulb. Simultaneous optical recordings from three different areas. Signals from three selected pixels are shown below the outline of the bulb. The approximate positions of these pixels are labeled with squares and numbers on the image of the bulb. The oscillation in the rostral region has a high frequency and relatively long latency and duration (detector 1 ). The oscillation from the middle region has a high frequency and short latency and duration (detector 2). The oscillation from the caudal region has a lower frequency and the longest latency (detector 3). The horizontal line labelled "10\% cineole" indicates the time of the command pulse to the odor solenoid. The data are filtered by digital high-pass $(5 \mathrm{~Hz})$ and low-pass $(30 \mathrm{~Hz})$ filters. For all of the figures the high-pass filter is an RC filter and the low-pass is Gaussian and all of the results are from single trials. The data in A were taken from Lam et al., (2000). B. Images of the input to the bulb as indicated by the signalsd from Calcium Green-1 dextran in the nerve terminals of the olfactory receptor axons. The input maps for isoamyl acetate and cineole are qualitatively different. The data in B were taken from Wachowiak et al., (2002).

Figure 2. Comparison of the rate of rise of the DC component elicited by isoamyl acetate and cineole. A. Typical example of the DC component elicited by $1.7 \%$ isoamyl acetate. The trace is the spatial average of five diodes in the middle region of the olfactory bulb. The DC component induced by isoamyl acetate has a relatively slow rise-time.

B. Multi-frame display of the spatial dynamics of the DC signal during the rising phase bracketed by red lines in A. The signal starts in a middle-caudal region and then spreads to almost the entire bulb. In B and D the fixed pseudocolor scale was used which represents the size of the signal. In this figure and in Figure 3 we show only the signal between the maximum and $60 \%$ of maximum so that the image of the bulb can also be seen. In all of the pseudocolor figures signals between 80 and $100 \%$ of the maximum are colored red.

C. Typical example from the same preparation of the DC component elicited by $1.7 \%$ cineole. The trace is the average of the same five selected diodes (used in A) in the middle region. The rise-time is much shorter.

D. The multi-frame display of the spatial dynamics of the DC component during the rising phase bracketed by red lines in $\mathrm{C}$. Again, the signal starts in the middle region and then spreads to almost the entire bulb. Arrowheads on the two traces $(A, C)$ designate where we assign the time of the start of the odor command pulse (red) and the start of the DC component (black) for latency analysis. The blue arrows indicate three cycles of the middle oscillation which rides on top of the DC signal. In this and subsequent figures the horizontal bar indicates the timing of the odor-command pulse. The traces are band-passed between 0.1 and $30 \mathrm{~Hz}$.

Figure 3. Comparison of the latencies of the middle oscillations elicited by isoamyl acetate and cineole. Same data as Figure 2 but digitally filtered with a $10 \mathrm{~Hz}$ high-pass RC filter.

A. Typical example of a middle oscillation elicited by $1.7 \%$ isoamyl acetate. The middle oscillation induced by isoamyl acetate had a relatively long latency.

B. Multi-frame display of the spatial dynamics of the oscillation during the cycle indicated by the red lines in A. 
The cycle initiates in a medial middle region and then spreads to the middle and caudal bulb. In B and D the fixed pseudocolor scale was used which represents the size of the signal. Because the size of the middle oscillation is relatively small, the pseudocolor images are noisier than those for the DC signal.

C. Typical example of the middle oscillation elicited by $1.7 \%$ cineole. The middle oscillation in response to cineole has a shorter latency.

D. Multi-frame display of the cycle indicated by the red lines in $\mathrm{B}$. The oscillation again begins in a medial middle region and then spreads to the middle and caudal bulb.

Arrowheads on the two traces $(\mathrm{A}, \mathrm{C})$ designate where we assigned the time of the first peak (black) and third peak (red) of the oscillation for the measurement of the latency and frequency. The traces are filtered at 10 to $30 \mathrm{~Hz}$. In this and subsequent figures the high-pass filter is an RC filter and the low-pass filter is a Gaussian.

Figure 4. The latency difference of the middle oscillation is not present in the rostral oscillation. The traces in $A$ are the spatial average of five diodes from the medial region of the bulb. The medial oscillation induced by cineole has a shorter latency than that induced by isoamyl acetate. On the other hand, the rostral oscillation elicited by cineole has similar latency (B) for the two odorants. The traces are filtered at 10 to $30 \mathrm{~Hz} .10 \%$ isoamyl acetate and $10 \%$ cineole were used. The arrows indicate the time we designated as the onset of the oscillation.

Figure 5. Comparisons of the envelopes of the rostral oscillations; three pairs including four different odorants from two preparations are illustrated. The envelopes are not obviously different even though the chemical structures and the human perception of the odorants are quite distinct. Each trace represents the average from 5-10 pixels. The data were filtered with a high-pass $\mathrm{RC}$ filter of $5 \mathrm{~Hz}$ to reduce the size of the $\mathrm{DC}$ components. A low pass Gaussian filter of $30 \mathrm{~Hz}$ was used to reduce high-frequency noise.

Figure 6. Comparisons of the envelopes of the middle and caudal oscillations; two pairs including two different odorants from two preparations are illustrated for the middle oscillation, two pairs including three different odorants from two preparations are illustrated for the caudal oscillation. The envelopes of the middle and caudal oscillation are not obviously different even thought the chemical structures and the human perception of the odorants are quite distinct. Each trace represents the average from 5-10 pixels. For the middle oscillation the data were filtered with a high-pass RC filter of $10 \mathrm{~Hz}$ to reduce the size of the DC components; a low pass Gaussian filter of $30 \mathrm{~Hz}$ was used to reduce high-frequency noise. For the caudal oscillation the data were filtered with a high-pass RC filter of $5 \mathrm{~Hz}$ to reduce the size of the DC components; a low pass Gaussian filter of $10 \mathrm{~Hz}$ was used to reduce high-frequency noise.

Figure 7. A. Comparison of the locations of the rostral oscillations in response to cineole, isoamyl acetate, and hexanone odor presentations at $0.3 \%$ of saturated vapor. The rostral oscillations are found in the same location for the three odorants. The time points shown are the time points of the largest signal over the largest area. The fixed pseudocolor scale was used which represents the size of the signal. B. Five time points during the cycles shown above for cineole and isoamyl acetate. The propagation in the rostral-caudal direction is similar for both odorants. The variable pseudocolor scale (see methods) was used which better represents the 
timing of the signals. C. Comparison of the locations of the middle oscillations in response to cineole and isoamyl acetate odor presentations at $15 \%$ of saturated vapor. The middle oscillations are found in the same location for the two different odorants. In these measurements the position of the image of the olfactory bulb differed from that shown in Figure 1 in that the rostral portion of the bulb was in the middle of the image. D: Comparison of the locations of the caudal oscillations in response to cineole and isoamyl acetate odor presentations at $10 \%$ of saturated vapor. In these examples the caudal oscillations are located in the same location for the two different odorants. In C. and D. the fixed pseudocolor scale was used which better represents the size of the signal. The time points shown are the time points of the largest signal over the largest area. 
Figure 1
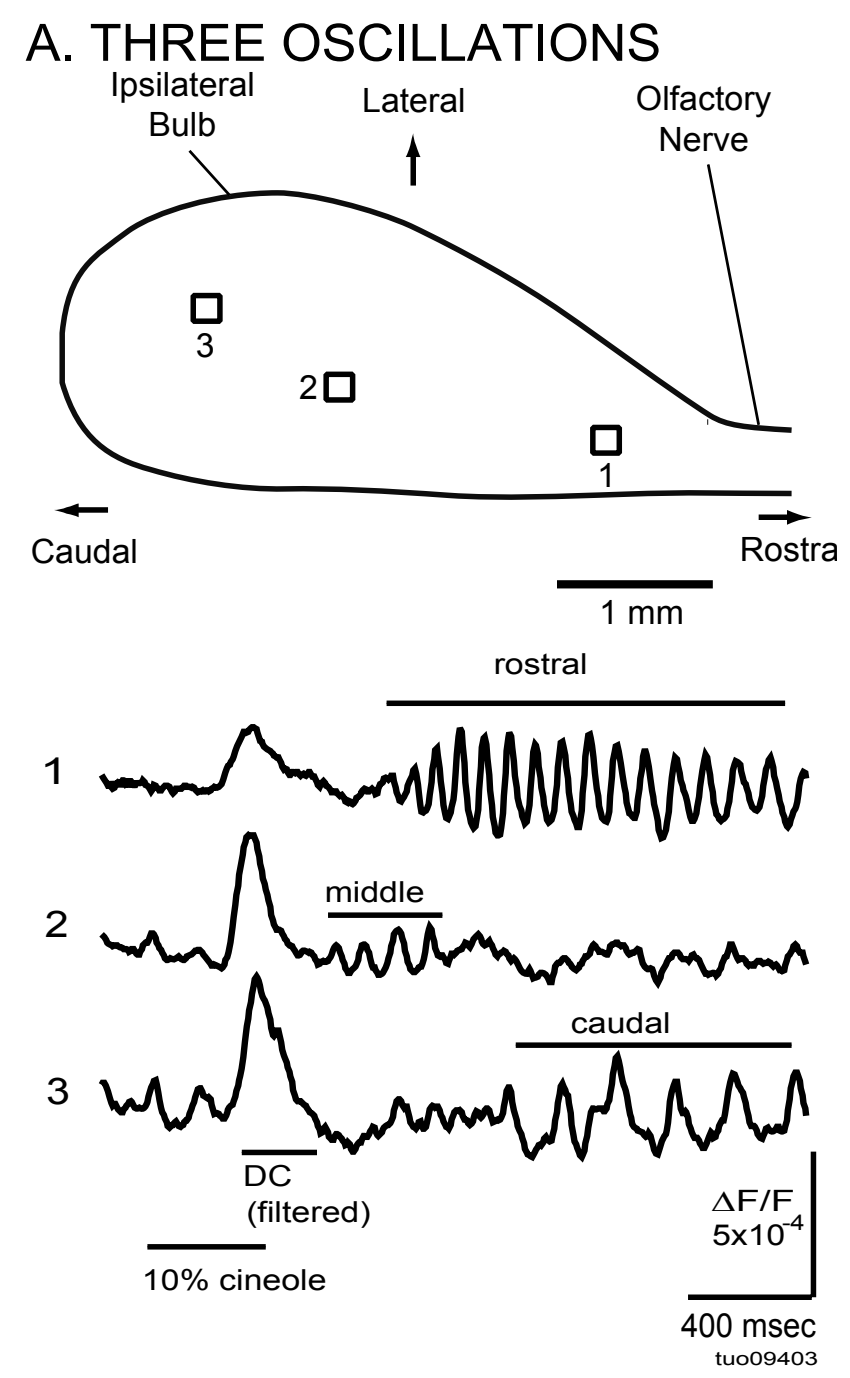

\section{B. INPUT TO THE BULB}
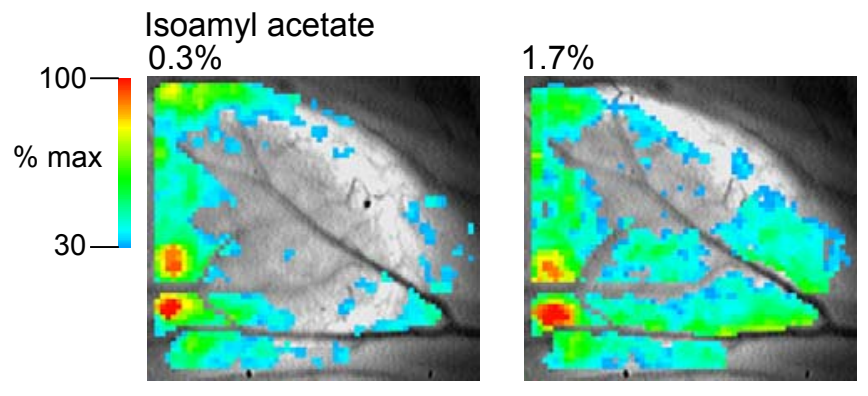

Cineole
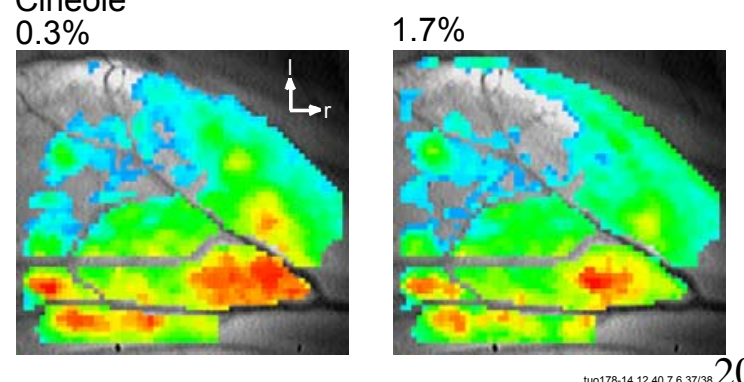
Figure 2

THE RATE OF RISE OF THE DC COMPONENT

(filtered: $0.1-30 \mathrm{~Hz}$ )

$1.7 \%$ isoamyl acetate

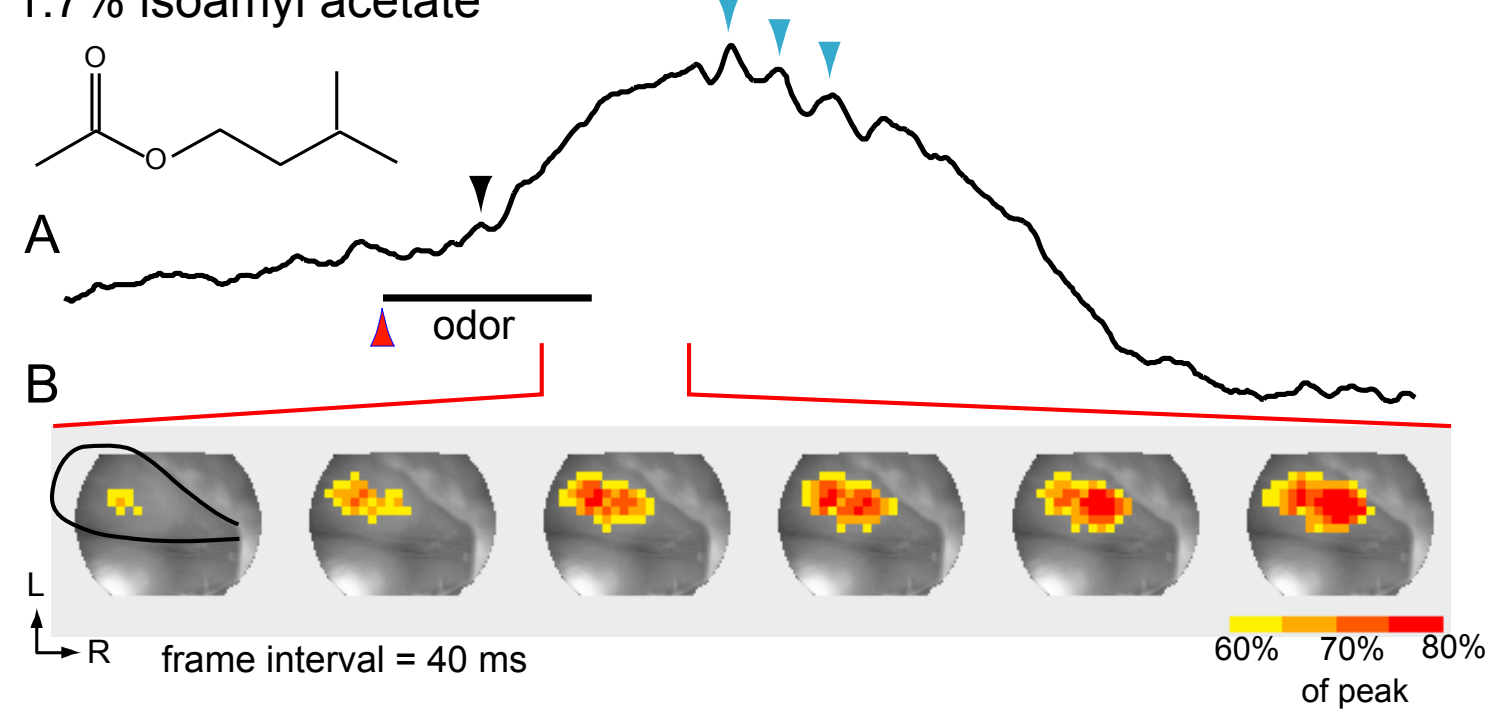

$1.7 \%$ cineole

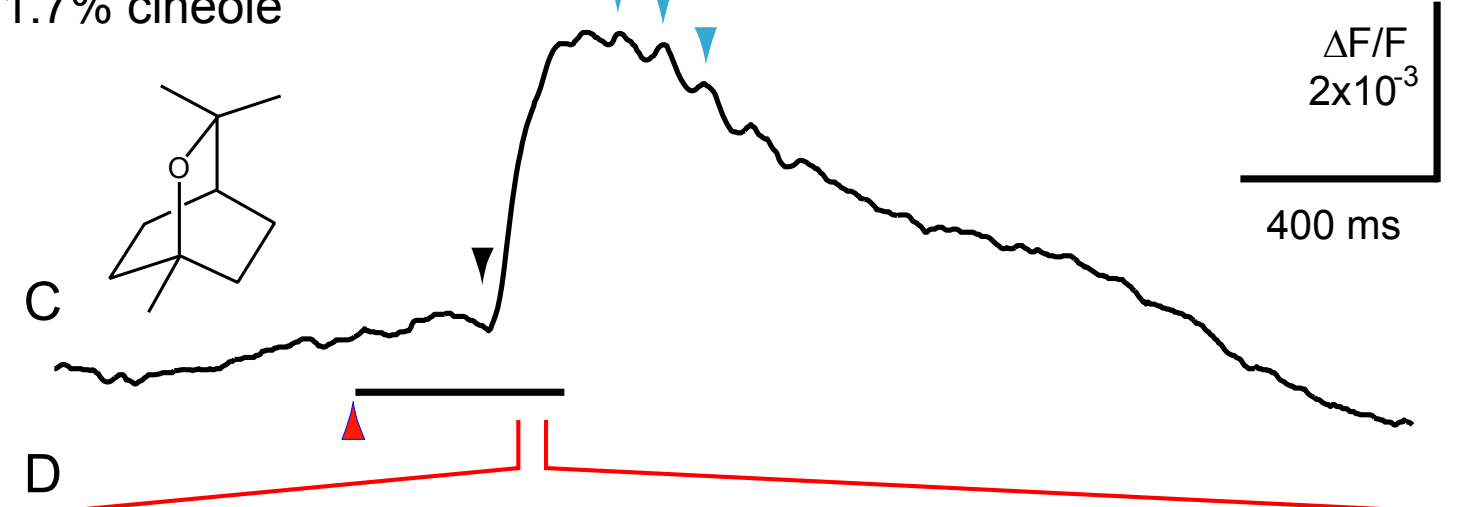

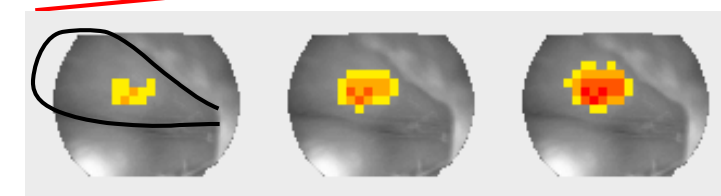

frame interval $=8 \mathrm{~ms}$

tuo09411-16

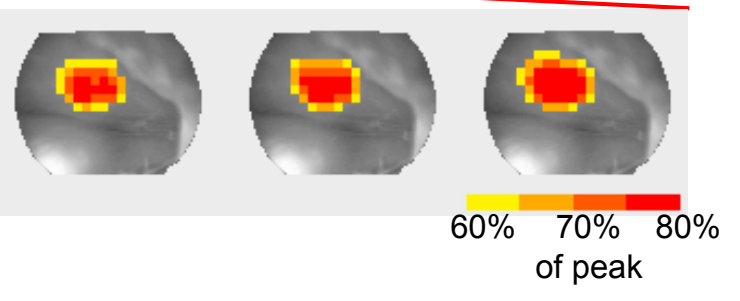


Figure 3

THE LATENCY OF THE MIDDLE OSCILLATION (filtered $10-30 \mathrm{~Hz}$ )

$1.7 \%$ isoamyl acetate

A
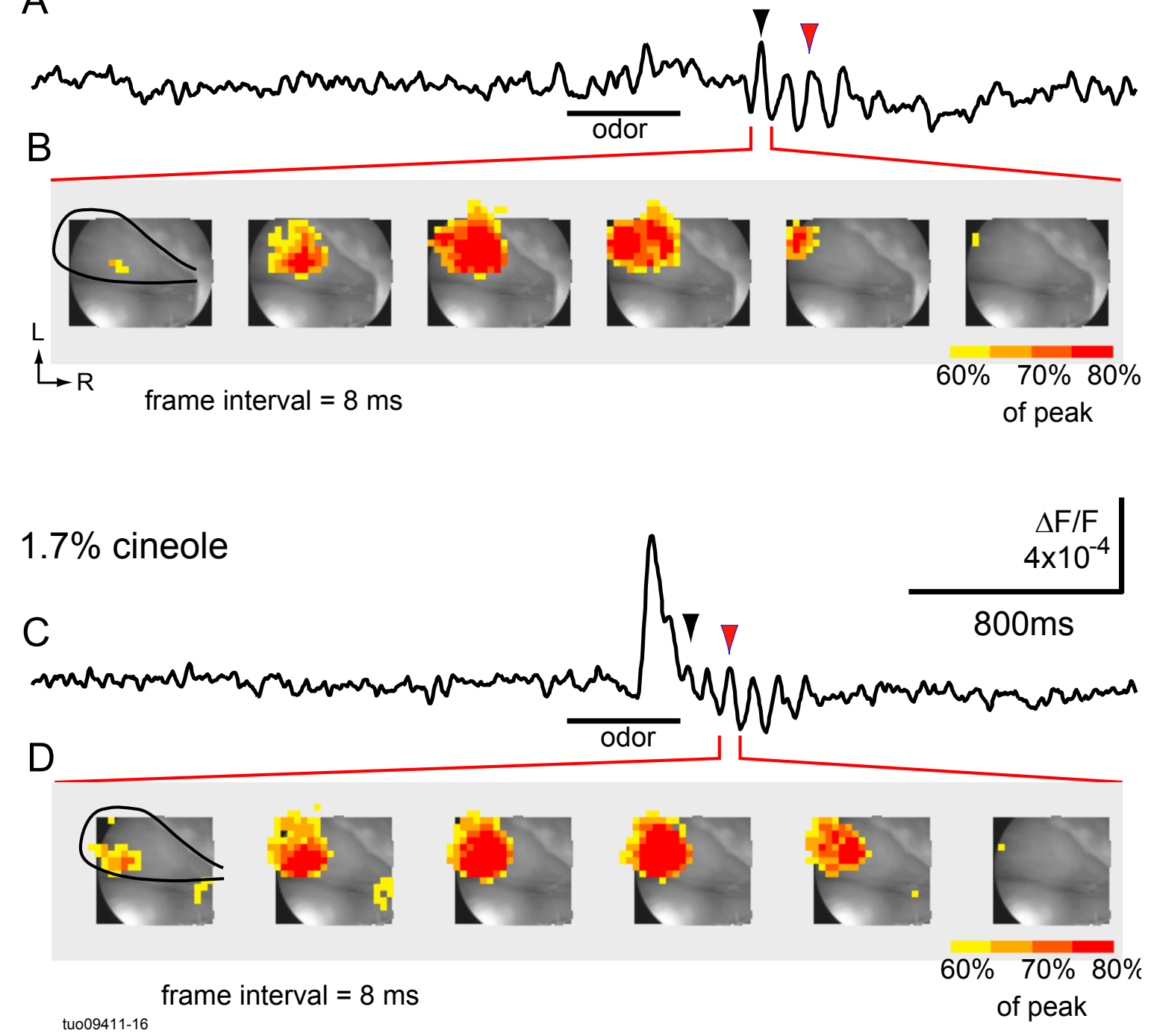
Figure 4

LATENCIES OF MIDDLE AND ROSTRAL OSCILLA (filtered: $10-30 \mathrm{~Hz}$ )

A. Middle region isoamyl acetate cineole \begin{tabular}{lr} 
& $\Delta \mathrm{F} / \mathrm{F}$ \\
B. Rostral region & $4 \times 10^{-4}$ \\
\hline
\end{tabular} isoamyl acetate $800 \mathrm{~ms}$

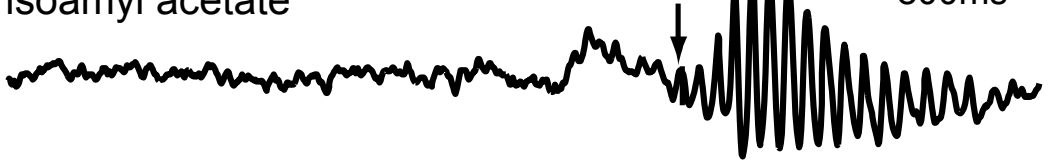
cineole 
Figure 5

ENVELOPES OF THE ROSTRAL OSCILLATION

$1.7 \%$ hexanone

调

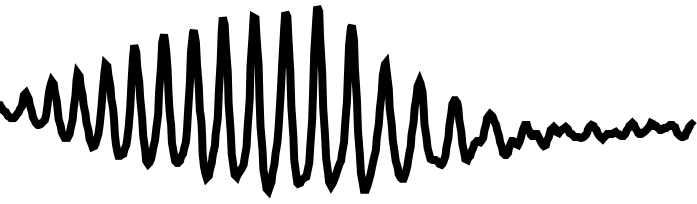

$1.7 \%$ isoamyl acetate

$\mathrm{N}^{\mathrm{O}}$

$\underset{4 \mathrm{~F} / \mathrm{F}}{4 \times 10^{-4}}$

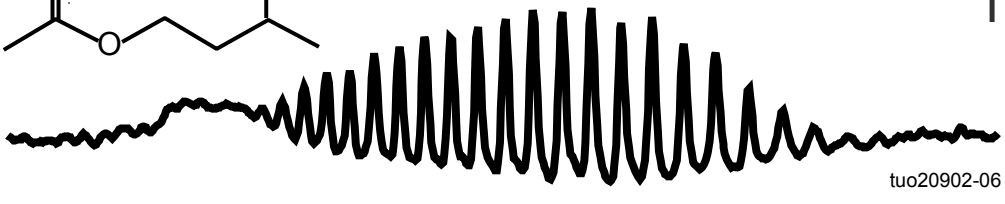

$500 \mathrm{msec}$

$0.3 \%$ cineole

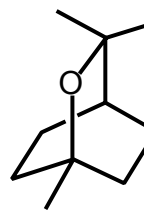

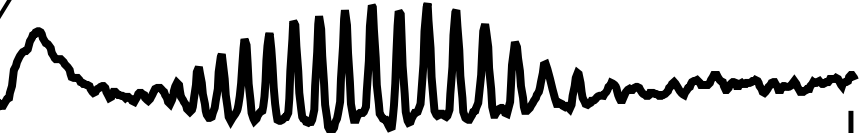

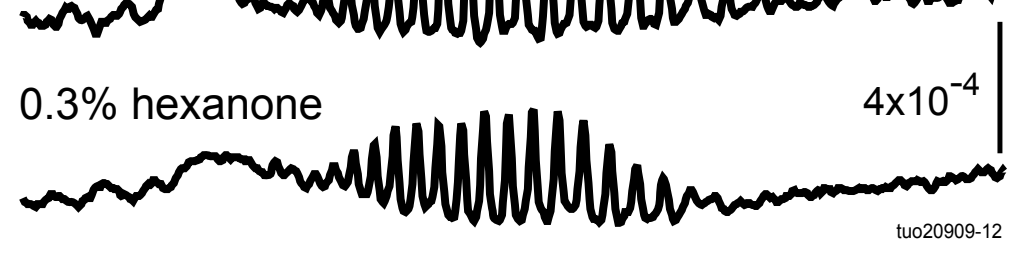

$500 \mathrm{msec}$

$6 \%$ isoamyl acetate

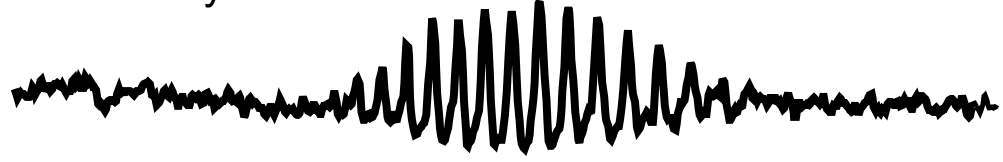

$6 \%$ hexanal

$7 \times 10^{-4}$

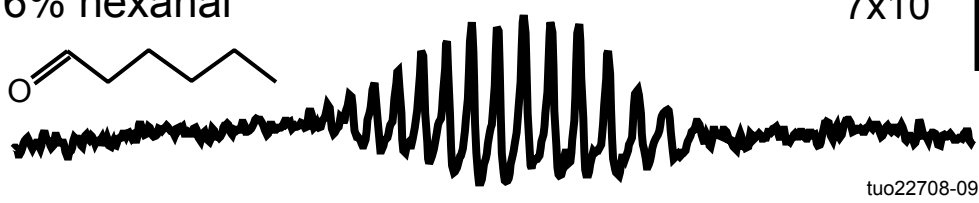

odor

$600 \mathrm{msec}$ 
FIGURE 6

ENVELOPES OF THE MIDDLE AND CAUDAL

OSCILLATION

MIDDLE

$15 \%$ cineole

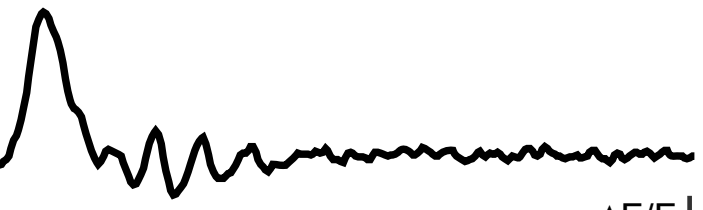

$\Delta \mathrm{F} / \mathrm{F}$

$15 \%$ isoamyl acetate

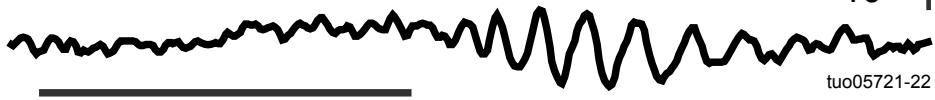

$400 \mathrm{msec}$

$6 \%$ cineole

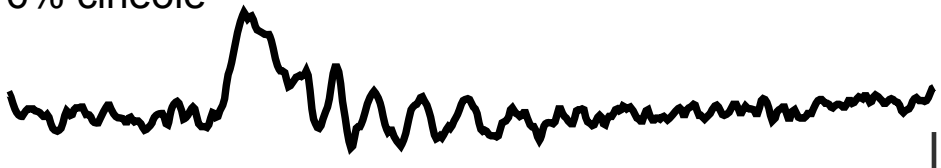

$6 \%$ isoamyl acetate

$3 \times 10^{-4}$

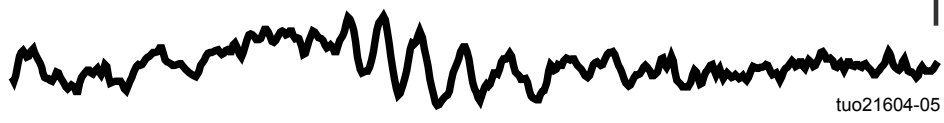

$400 \mathrm{msec}$

CAUDAL

$1.7 \%$ hexanone

Mmamman

$1.7 \%$ isoamyl acetate

$$
4 \times 10^{-4}
$$

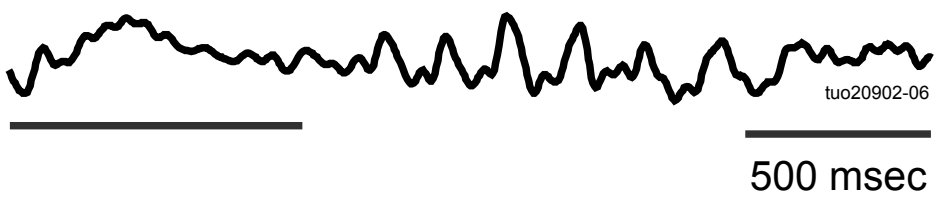

$6 \%$ cineole

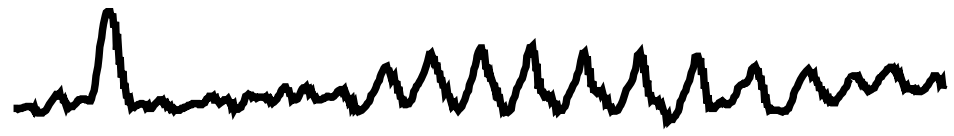

$6 \%$ isoamyl acetate $\quad 5 \times 10^{-4}$

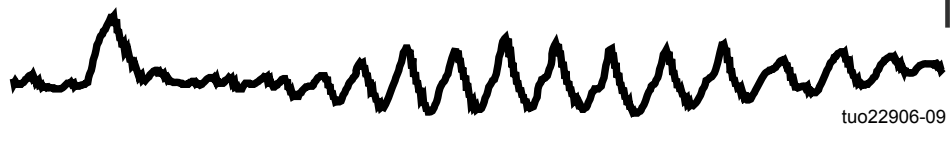

odor 
Figure 7

A. LOCATION OF THE ROSTRAL OSCILLATION

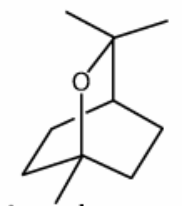

$0.3 \%$ cineole

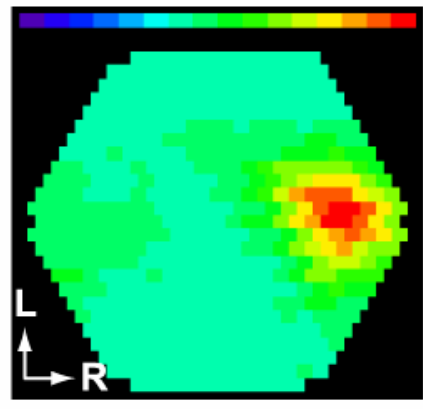<smiles>CC(=O)OCCC(C)C</smiles>

$0.3 \%$ isoamyl acetate

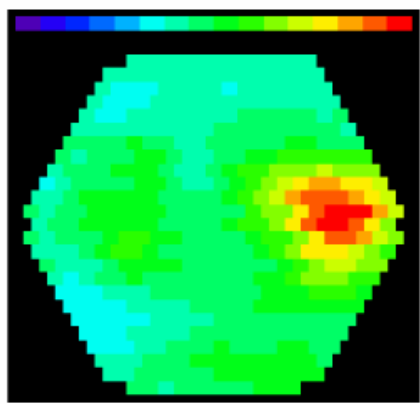<smiles>CCCCC(C)=O</smiles>

$0.3 \%$ hexanone

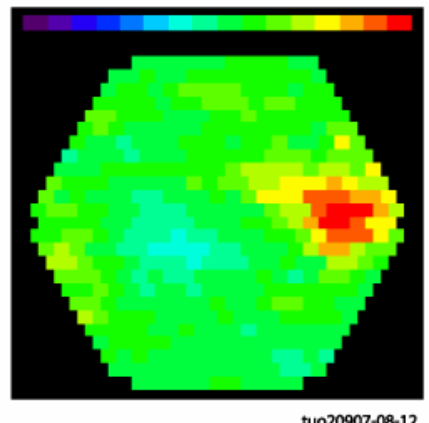

$1.0 \mathrm{~mm}$

B. PROPAGATION OF ONE CYCLE OF THE ROSTRAL OSCILLATION

$0.3 \%$ cineole

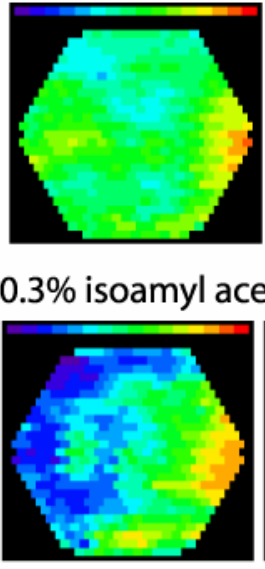

$0 \mathrm{~ms}$
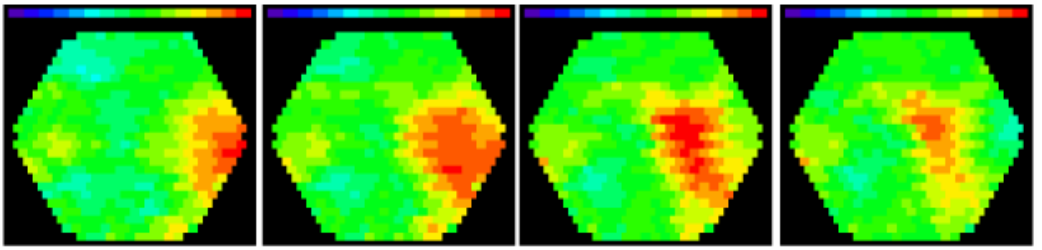

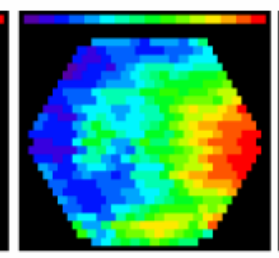

$4 \mathrm{~ms}$

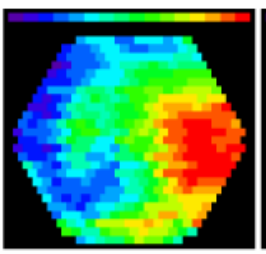

$8 \mathrm{~ms}$

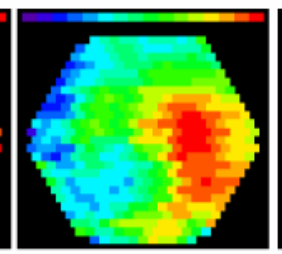

$12 \mathrm{~ms}$

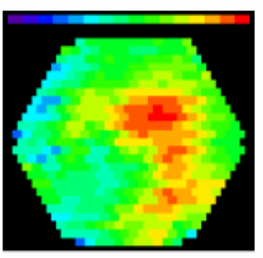

$16 \mathrm{~ms}$

\section{LOCATION OF MIDDLE OSCILLATION}

D. LOCATION OF CAUDAL OSCILLATION

$15 \%$ cineole

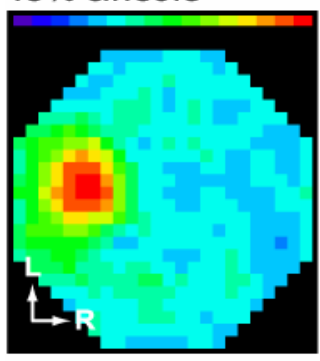
$15 \%$ isoamyl acetate

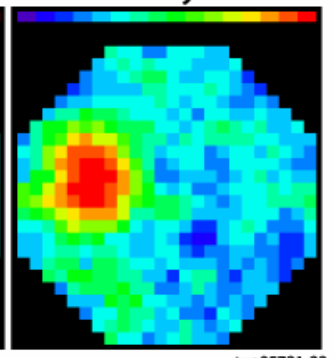

$10 \%$ cineole

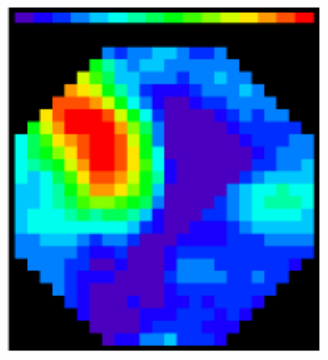
$10 \%$ isoamyl acetate

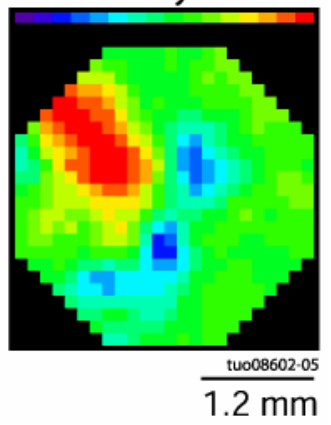

